\title{
Experimental Study of Twin Connected Pipe Jets
}

\author{
Shams Sourav, Ashraful Hossain Rifat, Muhammed Hasnain Kabir Nayeem, and Md. Abu Taher Ali
}

\begin{abstract}
Two parallel pipe jets of $20 \mathrm{~mm}$ diameter were placed $1.2 \mathrm{~mm}$ diameter apart and were connected by a $4 \mathrm{~mm}$ wide channel all along the central plane of the jets. The mean velocity flow field of the jets was investigated for three Reynolds number 16300, 34400 and 49200. The Reynolds number was based on the exit velocity of the jets and jet diameter. The experiment was performed in an air jet facility and yaw meter was used for measurement of mean velocity and its direction. Their variations along the longitudinal, transverse, and lateral directions have been analyzed. A significant change of the jet flow field is observed near the exit of the jet. The combined effect of the jets diminishes the presence of recirculation region at the immediate exit rather enhances the energy and momentum transfer between their individual flow fields. Static pressure and kinetic energy distribution are also studied and a momentous variations have been noticed with varying Reynolds number.
\end{abstract}

Index Terms - Twin Jets, Kinetic Energy, Mean Velocity, Static Pressure, Turbulence.

\section{INTRODUCTION}

Jet flow is one of the most significant phenomena in the field of engineering due to its numerous applications. Extensive research is going on different types of jet flow. Among them, twin jet has become one of the most widespread for the researchers for its improved mixing performance in various industrial application such as fuel injection for the combustion system in gas turbine engines, industrial burners, chemical reactors, cyclone separator etc. Interaction of twin jet and its turbulent characteristics has been studied for years. Earliest experiment on twin jet was done by Millar and Comings [6], where they reported two regions of sub-atmospheric and super-atmospheric static pressure field and negative pressure near the exit of the jet creates a converging curvature. Tanaka [8], [9] on his study identified three regions in the flow-field in the axial direction of the downstream direction. He defines them as converging, transition and combined regions respectively shown in Fig. 1, where converging region starts from jet exit

Published on December 30, 2020.

Shams Sourav, Department of Aeronautical Engineering, Military Institute of Science and Technology (MIST), Dhaka, Bangladesh.

(e-mail: sourav.shams ${ }^{@}$ gmail.com)

Ashraful Hossain Rifat, Department of Aeronautical Engineering,

Military Institute of Science and Technology (MIST), Dhaka,

Bangladesh.

(e-mail: rifat_hn@yahoo.com)

Muhammed Hasnain Kabir Nayeem, Department of Aeronautical Engineering, Military Institute of Science and Technology (MIST), Dhaka, Bangladesh

(e-mail: muhammednayeem753@gmail.com)

Md. Abu Taher Ali, Department of Aeronautical Engineering, Military Institute of Science and Technology (MIST), Dhaka, Bangladesh.

(e-mail: matali@ae.mist.ac.bd). to the point where inner shear layer of the jets merge. The transition region exists between the merge point and combined point. After the combined point the jet behaves as single jet. The interaction of two plane parallel jets was experimentally observed by Elbana et al. [10], where they found that mean velocity distribution of the parallel jets resembles the behavior of single jet after the combined point. Anderson and Spall [7]

experimentally investigated the flowfield of dual, parallel planar turbulent jet. Their results show that decay rates of mean velocity for the twin jet tend to a single jet result in the far flow field. Hamaili et al. [16] examined the influence of weak jet on strong jet numerically and found that weak jet attracts strong jet which tends to expand upto 30D to 40D where jet presents similar properties like single jet.

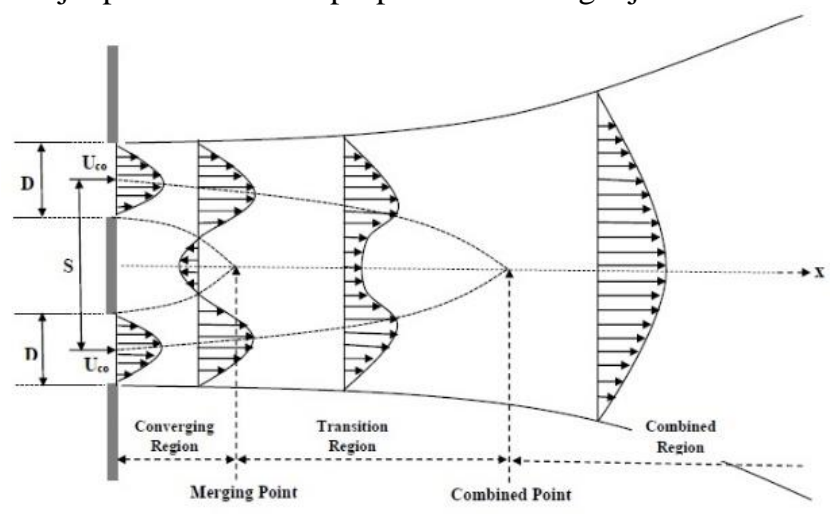

Fig. 1. Flow field of a twin jet.

Laban et al. [1] studied the effect of nozzle spacing for twin round jets emanating from sharp contraction nozzles by using particle image velocimetry (PIV). Results revealed that jets preserve their own properties initially, then interact and finally merge on the plane of symmetry at a certain distance from exit plane. Reduction of nozzle spacing enhance the mutual interaction of inner shear layers of two jets which causes them to merge earlier. Rahman and Tachie [14] experimentally studied the influence of Reynolds number on the flow field of twin jet where it is found that the merging region is sensitive to lower Reynolds number $(\operatorname{Re} \leq 3900)$ and nearly independent of higher Reynolds number. Lin and Sheu [17], [18] investigated twin jet by hot wire anemometry to observe that how mean velocity reaches self-similarity and their experiment shows that in the combined region of twin jet, the entrainment and spreading rate are greater than that of single jet. Zhao-gin et al. [19] experimentally studied the flow field characteristics in the mixing region of twin jet with varying jet spacing and Reynolds number. They obtained that interference between two jets increases with increased Reynolds number as well 
as reduced jet spacing and vice versa. Nasr and Lai [2]. [3] compared between two parallel and offset plane jets experimentally and later investigated the effect of jet spacing on the momentum flux for the combined point in stream wise direction. Mohapatra [15] numerically analyzed the flow field of two parallel plane jets using k- $\varepsilon$ model and identified the existence of negative and positive static pressure on both upstream and downstream of merging point. Erdem and Atli [4] investigated the effect of surface curvature by placing a cylindrical convex object between two rectangular jets and found that convex surface strongly deflects the jets towards the axis of symmetry as well as accelerates the flow field. Many other researchers [11]-[13] also studied twin jet both numerically and experimentally at different conditions.

In the present experimental study, a new geometry has been introduced by connecting two jets for smaller jet spacing. The idea of connecting two jets is to investigate the scenario that if the nozzle spacing is disturbed, how mean flow and turbulent characteristics change in the flow field for varying Reynolds number. The results are expected to enhance the development of further experimental and numerical approaches of twin connected jets.

\section{EXPERIMENTAL DETAILS}

The experimental was conducted in a circular air jet flow facility show in Fig. 2, which has an inlet diameter of $300 \mathrm{~mm}$ followed by an axial fan unit (consists of two axial fan), settling chamber, reduced, flow straightener and finally a discharge pipe nozzle which facilitates and axially symmetric twin jets of diameter $\mathrm{D}=20 \mathrm{~mm}$ and space ratio $\mathrm{S} / \mathrm{D}=1.2$. For connected twin jets, geometrically similar nozzle was used with connection length (L) and width (W) of $4 \mathrm{~mm}$ and $4 \mathrm{~mm}$ respectively.

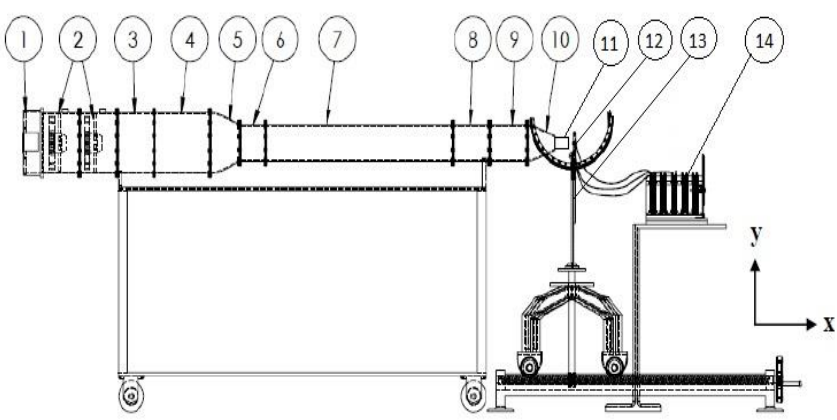

Fig.2.Air jet nozzle setup, (1) Flow Controller, (2) Fan Unit,

(3) GuideVane, (4) Settling Chamber, (5) Reducer, (6) Flow Straightener, (7) Flow Stabilizer Pipe, (8) Vortex Breaker,

(9) Discharge Pipe, (10) Reducer, (11) Discharge Nozzle, 12) Yaw meter, (13) Traversing Mechanism, 14) Pressure Transducer.

The geometry of the twin jet and twin connected jet nozzles are shown in Fig. 3. The jet setup has the capability of generating maximum flow velocity of $36 \mathrm{~m} / \mathrm{s}$ at the discharge nozzle exit. A flow damper is used at the inlet of the nozzle to regulate the flow. Experiments were performed for three different Reynolds numbers $(\mathrm{Re}=16300,34400$ and 49200) at a fixed twin jet connection length (L) and width $(\mathrm{W})$. To measure the flow velocity and direction, pressure probe method [5] was used by utilizing a calibrated three-hole wedge shaped yaw meter and three dual input differential pressure transducer of range $\pm 13.79 \mathrm{kPa}$ with 0.01 resolution and $\pm 0.3 \%$ accuracy. A three dimensional traversing mechanism was used to mount and move the probe at different longitudinal (x), transverse (y) and lateral (z) directions. Co-ordinate system of the nozzle is shown in Fig. 4. Ambient temperature and pressure were continuously observed and recorded during the experiment.

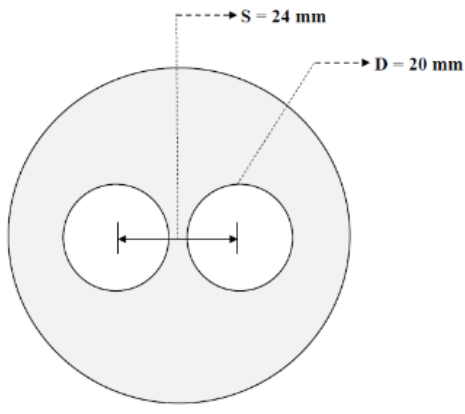

(a)

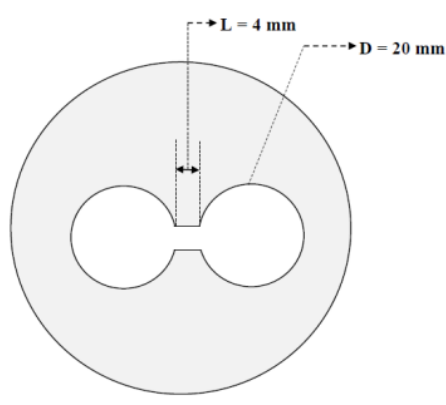

(b)

Fig. 3. Geometry of (a) Twin jet nozzle and (b) Twin connected jet nozzle.

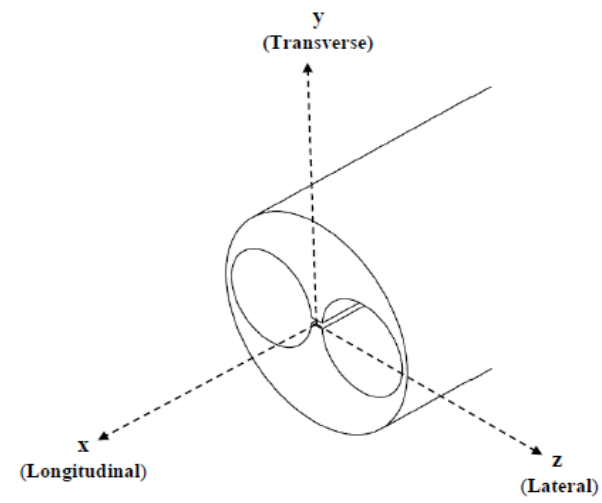

Fig. 4. Co-ordinate system of twin connected jets.

\section{RESULTS AND DISCUSSION}

\section{A. Centerline Mean Velocity Decay}

Fig. 5 shows the mean velocity (Ux) decay at the centerline of jet for three different Reynolds number $(\mathrm{Re})$ where the complete disappearance of recirculation zone is evident. Initially mean velocity decreases up to $\mathrm{X} / \mathrm{D}=2$ in the downstream direction and starts to increase again but not exceeding the velocity at the jet exit. In this region from $\mathrm{X} / \mathrm{D}=2$ to $\mathrm{X} / \mathrm{D}=5$, jets merge and interact where energy and momentum transfer occur. After $\mathrm{X} / \mathrm{D}=5$, jets combine and 
resembles the properties of a single jet. It is noticeable that length of the region where jets interact and merge in the near field is almost independent of the Reynolds number.

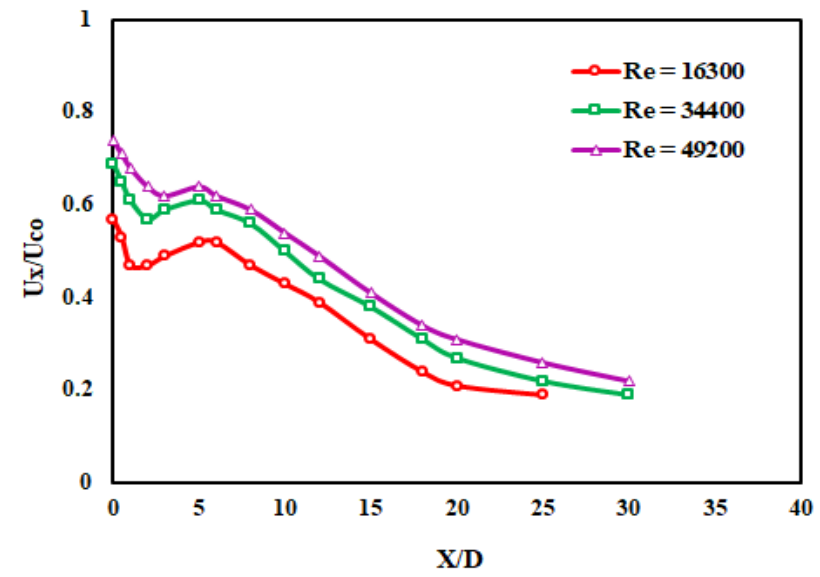

Fig. 5. Centerline mean velocity decay.

\section{B. Mean Velocity Profile in Transverse and Lateral Direction}

Mean velocity profile in the transverse direction is shown in Fig. 6. Which exhibits a rapid drop in the near field for all three Reynolds number. This sudden pick and drop can be caused by increased static pressure in the inner shear layer of the merging region. It is also observed that at low Reynolds number, mean velocity decreases is steeper than that of high Reynolds number. But in the far field due to entrainment of ambient fluid, jet velocity reduces at slower rate which indicates that twin connectedjets exhibit greater turbulent characteristics in low Reynolds number.

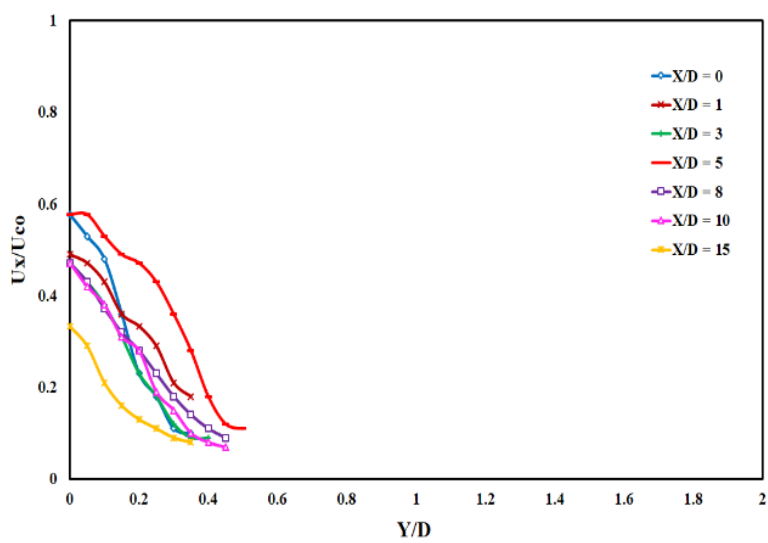

(a)

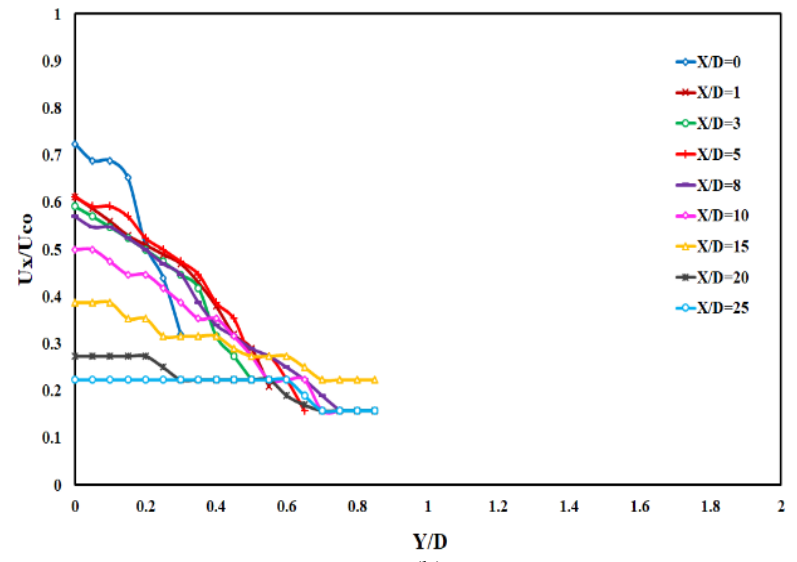

(b)

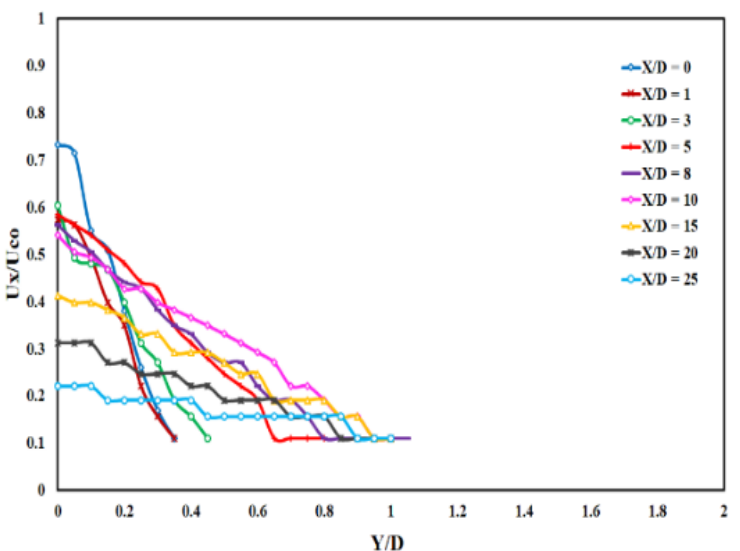

(c)

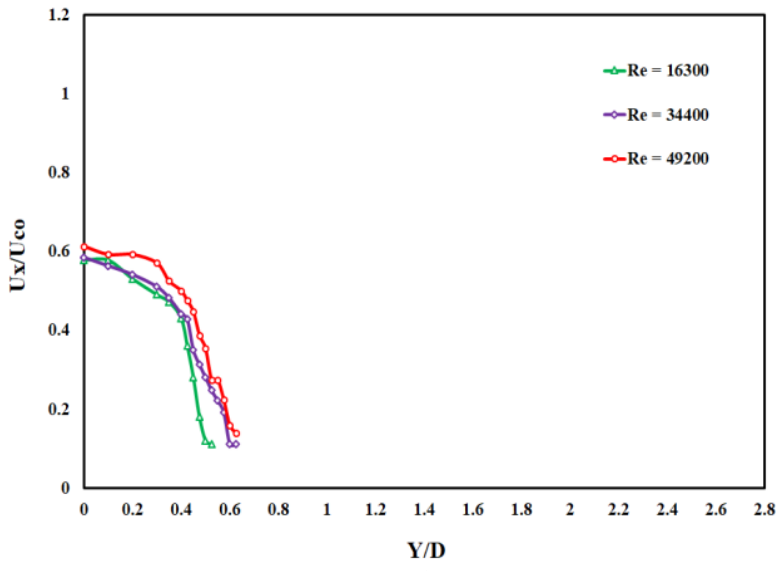

(d)

Fig. 6. Mean velocity profile in transverse direction at (a) $\mathrm{Re}=16300$, (b) $R e=34400$, (c) $R e=49200$, (d) different $R e$ for $X / D=3$.

Fig. 7. Represents the mean velocity profile at lateral direction and it distinctively shows the symmetric property with reference to $x-y$ plane. In the near field velocity picks have been observed up to $\mathrm{X} / \mathrm{D}=5$ and diminished in the far field afterwards with the entrainment of surrounding fluid. Pick velocity also shifted towards $x-y$ plane with the downstream in the near field for all three Reynolds number which is caused by the attraction and interaction between the jet shear layers. It is remarkable that jet width decreases as Reynolds number increases.

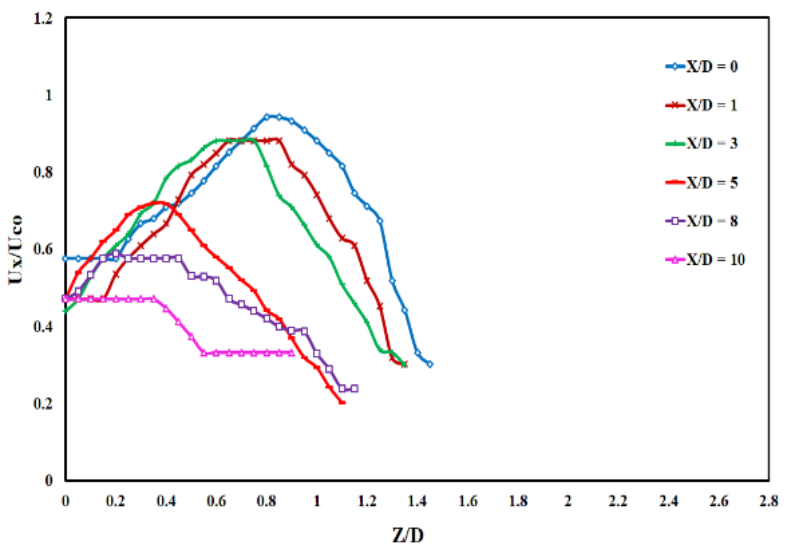

(a) 


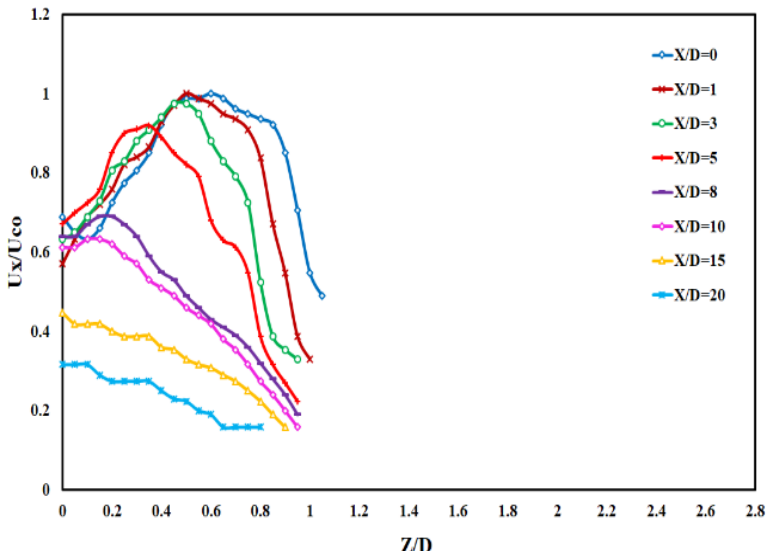

(b)

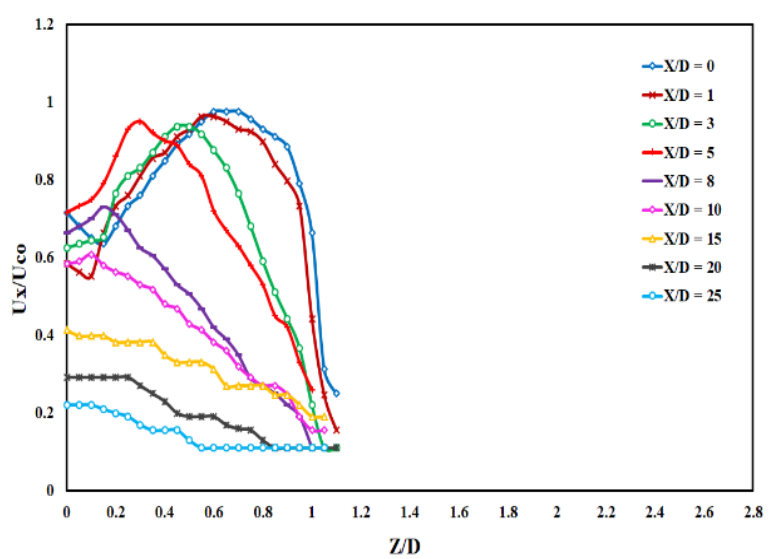

(c)

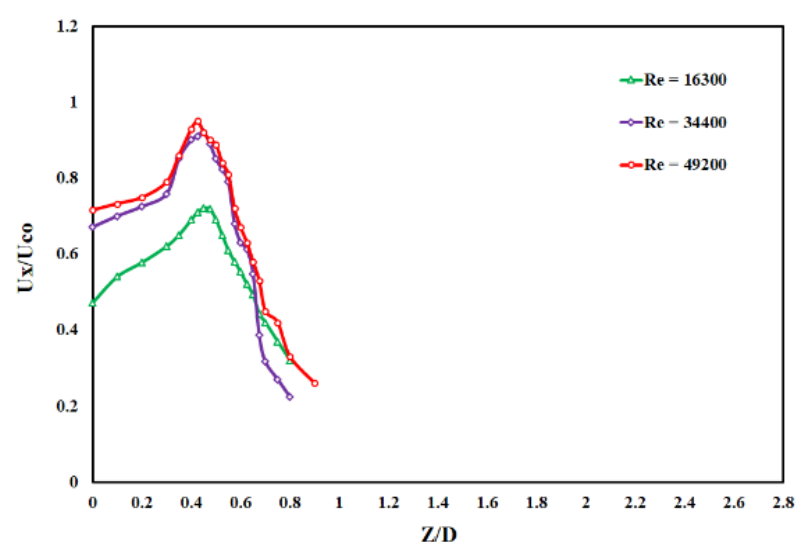

(d)

Fig. 7. Mean velocity profile in lateral direction at (a) $\mathrm{Re}=16300$, (b) $\mathrm{Re}=34400$, (c) $\mathrm{Re}=49200$, (d) different $\mathrm{Re}$ for $\mathrm{X} / \mathrm{D}=5$.

\section{Static Pressure Distribution}

The static pressure (Ps) distribution along the centerline of twin connected jets is shown by Fig. 8. Due to disappearance of recirculation region, negative pressure does not exist at immediate exit of the jet. Interaction and merging of inner shear layers exhibits increased static pressure in the upstream region. Entrainment of ambient fluid helps static pressure to decay towards atmosphere in further downstream direction after $\mathrm{X} / \mathrm{D}=3$. It is also evident that higher the Reynolds number, lower the static pressure in the near filed of the jet and static pressure drops towards atmosphere earlier if Reynolds number increases which represents twin connected jet has more turbulent instability at lower Reynolds number.

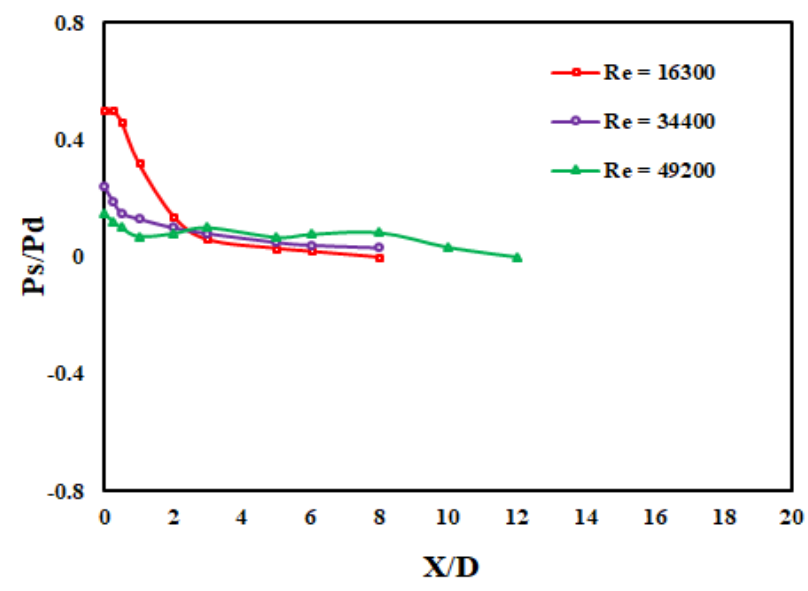

Fig. 8.Static pressure distribution.

\section{Mean Kinetic Energy Distribution}

Fig. 9 represent the mean kinetic energy $(\mathrm{K})$ distribution along the centerline of the jet flow field where gradual decrease of energy is noticed in the near field up to $\mathrm{X} / \mathrm{D}=$ 1.5 (approx.) in the further downstream, energy increases at certain level upto $\mathrm{X} / \mathrm{D}=6$ and starts decreasing again where jet resembles the properties of a single jet. It indicates that after merging point, connection of twin jet accelerates the kinetic energy and momentum transfer in the flow field. As Re increases, this energy and momentum transfer also increases.

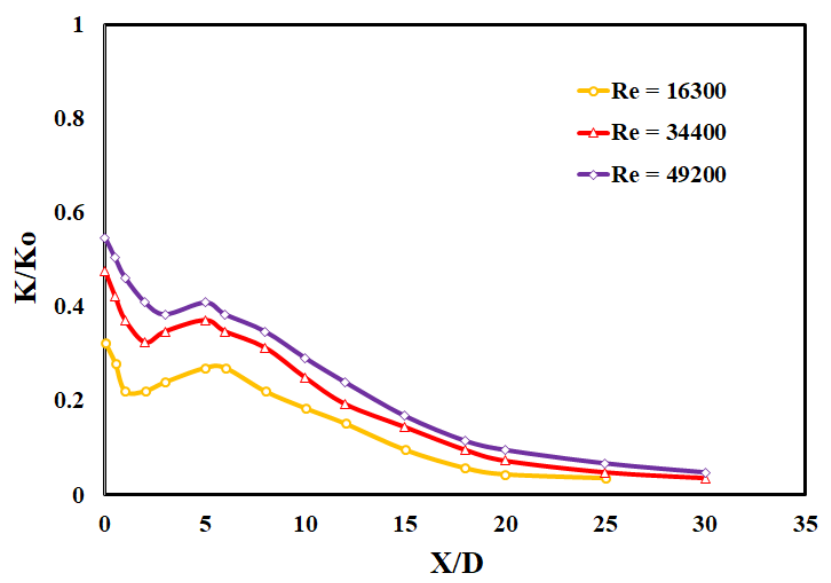

Fig. 9. Mean kinetic energy distribution.

\section{CONCLUSION}

Effect of connecting twin jets at a smaller space ratio of $\mathrm{S} / \mathrm{D}=1.2$ is investigated in this experiment at three different Reynolds number ( $R e=16300,34400$ and 49200). The results show that is has significant impact on the flow field of twin jets. It not only abolishes the recirculation region but also enhances the interaction and merging of two jets in the near field up to $\mathrm{X} / \mathrm{D}=5$. Region wise centerline mean velocity decay is nearly independent of Reynolds number and jet resembles the properties of a single jet in the far field. Furthermore, sudden rise and fall of mean velocity is evident in the near field of transverse direction due to high momentum and energy transfer during interaction of inner shear layers. The results also exhibit symmetry in the flow 
field at lateral direction if $\mathrm{x}-\mathrm{y}$ plane is considered as central plane and is this direction jet width significantly reduces when Reynolds number increases. At the immediate exit of the nozzle (upto $\mathrm{X} / \mathrm{D}=5$ ), lower Reynolds number has tendency to show greater turbulent behavior than higher Reynolds number. When static pressure distribution was observed along the centerline in longitudinal direction, no negative pressure was existent due to absence of recirculation region and static pressure drops at this region as Reynolds number increases.

\section{ACKNOWLEDGMENT}

Authors would like to acknowledge the contribution of previous researchers for the development of whole test facility. They are also grateful to the department of aeronautical engineering as well as all the staffs of aerodynamics and machine lab for their utmost cooperations.

\section{REFERENCES}

[1] A. Laban, S. S. Aleyasin, M. F. Tachie and M. Koupriyanov, "Experimental investigation ofnozzle spacing effects on characteristics of round twin free jets", Journal of Fluids Engineering, vol.121, 071201-1, July, 2019.

[2] A. Nasr, and J. C. S. Lai, "Comparison of flow characteristics in the near field of two parallel plane jets and an offset plane jet," Phys. Fluids, vol.9, no.10, pp.2919-2931, June, 1997.

[3] A. Nasr, and J. C. S. Lai, "A turbulent plane offset jet with small offset ratio,' Exp. Fluids, vol.24, pp.47-57, January, 1998.

[4] D. Erdem and V. Atli, "Interaction of two parallel Rectangular Jets", Proceedings of $23^{\text {rd }}$ ICAS Congress, Toronto, Canada,September, 2002.

[5] D. W. Bryer andR. C. Pankhurst, "Pressure probe methods for determining wind speed and flow direction," Published by National Physical Laboratory, London, 1971.

[6] D. R. Miller and E. W. Comings, "Force momentum fields in a dual jet flow,'’ J. Fluid Mech., vol. 7, pp.237-256, February, 1960.

[7] E. A. Anderson and R. E. Spall, "Experimental and numerical investigation of two-dimensional parallel jets', Journal of fluids engineering, vol.123, pp.401-406, June, 2001.

[8] E. Tanaka, "The interference of two-dimensional parallel jets -1 st report, experiments on dual jet'" Bull. JSME, vol.13, no. 56, pp.272$280,1970$.

[9] E. Tanaka, "The interference of two-dimensional parallel jets -2nd report, experiments on the combined flow of dual jet," Bull. JSME, vol.17, no.109, July, pp.920-927, 1974.

[10] H. Elbanna, S. Gahin and M. I. I. Rashed, "Investigation of two plane parallel jets,'’AIAA Journal, vol.21, no. 7, pp. 986-990, July, 1983.

[11] K. Murai, M. Taga and K. Akagawa, "An experimental study on confluence of two dimensional jets," Bull. JSME, vol.19, pp.956-964, February, 1976.

[12] M. A. Azim, "Characteristics of twin axisymmetric free jets", Proceedings of $8^{\text {th }}$ International Conference on Mechanical Engineering, Dhaka, Bangladesh, December, 2009.

[13] M. V. Philippov, I. A. Chokhar, and V. V. Terekhov, "Experimental study of interaction of two parallel circular jets," J. Phys. Conf. Ser. 1359:012019, 2019.

[14] M. S. Rahman and M. F. Tachie, "Reynolds number effect on flow characteristics of surface single and twin jets", Journal of Hydraulic Research, vol.57, no.6, pp.808-821, November, 2018.

[15] R. C. Mohapatra, "Prediction on turbulent flow of two parallel plane jets using k- $\varepsilon$ model”, Journal of Open Access Library, vol.4, e3291, February, 2017.

[16] S. L. Hamaili, A. Mataoui and M. Aksouh, "Effect of a weak jet on a strong jet”, journal of applied fluid mechnaics, vol.12, no.6, pp.20832093, February, 2018.

[17] Y. F. Lin and M. J. Sheu, "Investigation of two plane parallel unventilated jets,'’ Exp. Fluids, vol.10, pp.17-22, October, 1990.

[18] Y. F. Lin and M. J. Sheu, "Interaction of Parallel Turbulent Plane Jets,’’ AIAA J., vol.29, pp.1372-1373, September, 1991.
[19] Y. Zhao-gin, Z. H. Jun and L. J. Jong, "Experimental study on flow field characteristics in the mixing region of twin jets." Journal of Hydrodynamics: Ser. B, vol.19, no.3, pp.309-313, June, 2000

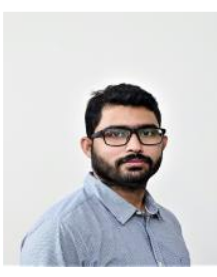

Shams Sourav is an aircraft engineer and pursuing his MSc. from the Department of Aeronautical Engineering in Military Institute of Science and Technology (MIST), Dhaka, Bangladesh. He completed his BSc in Aeronautical Engineering from MIST in 2014.

He has over 5 years of professional experience in aviation and is actively involved in research in the field of fluid mechanics and aerodynamics.

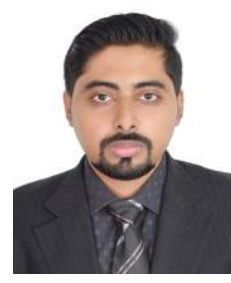

Ashraful Hossain Rifat is a professional aircraft engineer currently pursuing his MSc. from Department of Aeronautical Engineering in Military Institute of Science and Technology (MIST), Dhaka, Bangladesh.He completed his BSc in Aeronautical Engineering from MIST in 2014.

He has more than 5 years of experience in aircraft maintenance engineering with active research skill in the field of fluid mechanics.

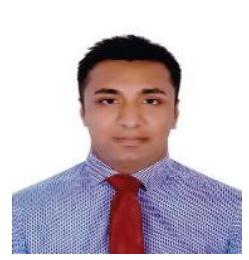

Muhammed Hasnain Kabir Nayeem is pursuing his M.Sc. from Department of Aeronautical Engineering (AE) in Military Institute of Science and Technology (MIST), Dhaka, Bangladesh. He completed his BSc in Aeronautical Engineering from MIST, Dhaka Bangladesh.

$\mathrm{He}$ has 2 years of research experience as a research assistant in Computer Science and Engineering Department, MIST. One of his published books named as title: "Study of circular swirling jet" published by LAP lambert academic publishing, ISBN-978-620-028036-7.

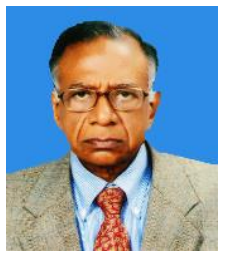

Md. Abu Taher Ali is a professor of Department of Aeronautical Engineering (AE) in Military Institute of Science and Technology (MIST), Dhaka, Bangladesh. He did his $\mathrm{PhD}$ in Mechanical Engineering from Imperial college, University of London, England (1980). He pursued his Masters of Science in Mechanical Engineering from Bangladesh University of Engineering and Technology, Dhaka, Bangladesh (1972). He completed his Bachelor of Engineering in Mechanical Engineering with $1^{\text {st }}$ class, $4^{\text {th }}$ positionfrom East Pakistan University of Engineering and Technology, Dhaka (1966).

$\mathrm{He}$ has over 50 years of teaching experience in the corresponding department. 